\title{
QTIPS: A Novel Method of Unsupervised Determination of Isotopic Amino Acid Distribution in SILAC Experiments
}

\author{
David J. Dilworth, Ramsey A. Saleem, Richard S. Rogers, \\ Hamid Mirzaei, John Boyle, and John D. Aitchison \\ Institute for Systems Biology, Seattle, Washington, USA
}

Stable incorporation of labeled amino acids in cell culture is a simple approach to label proteins in vivo for mass spectrometric quantification. Full incorporation of isotopically heavy amino acids facilitates accurate quantification of proteins from different cultures, yet analysis methods for determination of incorporation are cumbersome and time-consuming. We present QTIPS, Quantification by Total Identified Peptides for SILAC, a straightforward, accurate method to determine the level of heavy amino acid incorporation throughout a population of peptides detected by mass spectrometry. Using QTIPS, we show that the incorporation of heavy amino acids in baker's yeast is unaffected by the use of prototrophic strains, indicating that auxotrophy is not a requirement for SILAC experiments in this organism. This method has general utility for multiple applications where isotopic labeling is used for quantification in mass spectrometry. (J Am Soc Mass Spectrom 2010, 21, 1417-1422) (C) 2010 American Society for Mass Spectrometry

Q uantitative mass spectrometry analyses have revolutionized the ability to interrogate the proteome. The stable incorporation of labeled amino acids in cell culture (SILAC) [1] is a common method for quantitatively assaying proteomes in many systems and identifying functionally relevant, conditionspecific sub-proteomes [2-4]. The SILAC method utilizes cells cultured in the presence of isotopically normal or heavy amino acids enabling relative quantitative proteomics of multiple experimental conditions.

While the use of SILAC labeling to quantify proteomic differences between samples does not necessitate the complete incorporation of heavy labels, incomplete incorporation confounds downstream analysis. Long-term growth in SILAC media would help ensure complete incorporation, but it is not economically practical and is problematic because of time-dependent arginine-to-proline interconversion [5-7], which is readily detected in yeast after long-term culturing in SILAC media [8]. Efficient label incorporation and the total growth time in SILAC media are therefore, important considerations that must be balanced to achieve the greatest label efficiency in the minimum amount of time.

Several methods of determination of SILAC incorporation have been described $[1,6,9,10]$. Often, estimates of incorporation are based on manually curated data for a limited number of proteins and/or peptides, which may introduce artifacts resulting from variable protein

Address reprint requests to Dr. J. D. Aitchison, Institute for Systems Biology, Aitchison Laboratory, 1441 N 34th Street, Seattle, WA 98103, USA. E-mail: jaitchison@systemsbiology.org turnover rates [11, 12]. We hypothesized that the global level of SILAC incorporation could be directly and accurately inferred by comparing the number of heavy peptide identifications to all peptide identifications, both heavy and light, in a given protein sample for a given heavy labeled amino acid. Such a method would be advantageous as it would require only routine shotgun MS/MS analysis and obviate the need for area under the curve (AUC) analysis of the MS1 trace (Supplemental Figure, which can be found in the electronic version of this article). We used the technique described herein to test parameters in the yeast Saccharomyces cerevisiae, exploring the number of doubling times required for incorporation of isotopically heavy amino acids and the effect of intact versus disabled amino acid biosynthesis pathways.

\section{Material and Methods}

\section{Yeast strains, Growth Conditions, and Sampling}

All experiments were performed using the Saccharomyces

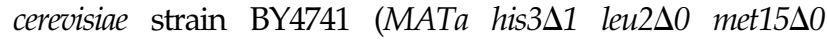
ura $3 \Delta 0$ ) and a previously described derivative of BY4742 auxotrophic for both lysine and arginine [13]. Complete synthetic growth media, CSM, [20 g/L D-glucose (VWR, West Chester, PA, USA), $0.67 \mathrm{~g} / \mathrm{L}$ Complete Supplement Mixture minus arginine, lysine, and histidine (Sunrise Science Products, San Diego, CA, USA), $1.7 \mathrm{~g} / \mathrm{L}$ Difco yeast nitrogen base $\mathrm{w} / \mathrm{o}$ amino acids and ammonium sulfate (BD, Franklin Lakes, NJ, USA), 5 g/L ammonium sulfate (Fisher, Pittsburgh, PA, USA), $30 \mathrm{mg}$ / L L-Histidine (Mallinckrodt Baker, Phillipsburg, 
NJ, USA), 45 mg/L L-Lysine (Fisher, Pittsburgh, PA, USA) and $50 \mathrm{mg} / \mathrm{L}$ L-arginine (Fisher, Pittsburgh, PA, USA)] was used for standard, light-labeled preparations. Heavy preparations were obtained by growth in SILAC-KR complete synthetic media (identical to standard CSM formulation above, except that L-Lysine${ }^{13} \mathrm{C}_{6},{ }^{15} \mathrm{~N}_{2} \cdot \mathrm{HCl}$ (Sigma-Aldrich, St. Louis, MO, USA) and L-Arginine $-{ }^{13} \mathrm{C}_{6},{ }^{15} \mathrm{~N}_{4} \cdot \mathrm{HCl}$ (Sigma-Aldrich, St. Louis, MO, USA) were included in place of their isotopically light variants. All samples were obtained from $\log$ phase cultures grown at $30^{\circ} \mathrm{C}$ with shaking at 200 $\mathrm{rpm}$ at the indicated generation number based on the empirically derived $105 \mathrm{~min}$ doubling time. For each sample, $\sim 1 \times 10^{8}$ cells were harvested in $50 \mathrm{~mL}$ conical tubes by centrifugation at $7000 \times \mathrm{g}$ for $5 \mathrm{~min}$, washed with $1 \mathrm{~mL}$ of sterile water in a $1.7 \mathrm{~mL}$ tube, centrifuged for $15 \mathrm{~s}$ at $10,000 \times g$ and the cell pellet was frozen in liquid nitrogen and stored at $-80^{\circ} \mathrm{C}$.

\section{Yeast Whole Cell Lysate Preparation, Trypsin Digestion, and Sample Clean-Up for MS/MS Analysis}

Cell pellets were resuspended in $500 \mu \mathrm{L}$ of $100 \mathrm{mM}$ ammonium bicarbonate, $\mathrm{pH} \mathrm{9,} \mathrm{and} 300 \mu \mathrm{L}$ of acidwashed glass beads (425-600 $\mu \mathrm{m}$, Sigma-Aldrich, St. Louis, MO, USA) were added. Tubes were mixed vigorously for $10 \mathrm{~min}$ at $4^{\circ} \mathrm{C}$ and cellular debris was removed by centrifugation at $15,000 \times g$ for $5 \mathrm{~min}$ at $4{ }^{\circ} \mathrm{C}$. The supernatants were transferred to fresh $1.7 \mathrm{~mL}$ centrifuge tubes and the protein concentration was determined for each lysate using the Warburg-Christian equation: protein concentration $(\mathrm{mg} / \mathrm{mL})=1.55$. $\mathrm{A}_{280}-0.76 \cdot \mathrm{A}_{260}$. We have performed QTIPS analyses on data obtained by shotgun MS/MS using as little as 5 $\mu \mathrm{g}$ of total yeast cell lysate. In general, $50 \mu \mathrm{g}$ of protein was brought to $100 \mu \mathrm{L}$ with $100 \mathrm{mM}$ ammonium bicarbonate, $\mathrm{pH} 9$. The sample was reduced with tris(2carboxyethyl)phosphine, alkylated with iodoacetamide and then digested with sequencing grade modified porcine trypsin (Promega, Madison, WI, USA). Digested samples were desalted using Vydac C18 Silica MicroSpin columns (The Nest Group, Southborough, MA, USA) by manufacturer's instructions. Purified peptides were diluted in load buffer $(0.1 \%$ formic acid, vol/vol) before MS/MS analysis.

\section{Mass Spectrometry Runs and Pipeline Processing}

Mass spectrometry was performed on Thermo Electron LTQ and LTQ-Orbitrap mass spectrometers equipped with an electrospray ionization source and an Agilent HP1100 liquid chromatography system. The gradient was run from 10 to $35 \%$ acetonitrile over $60 \mathrm{~min}$ at a constant flow rate of $0.350 \mu \mathrm{L} / \mathrm{min}$. XCalibur Raw files were converted to mzXML format using ReAdW (ver. 4.2.0) using the readw profile and default parameters. Spectral searches were done for tryptic fragments using X!Tandem [14] (ver. 2007.07.01.3 with the k-score plugin
[15]. Searches were done against a non-redundant $S$. cerevisiae reference protein database (the union of the SGD, Ensembl, NCI, and GenBank databases, plus keratin and trypsin) containing 13,616 entries. Parent tolerance was $\pm 3 \mathrm{Da}$, the fragment mass tolerance was $\pm 0.4 \mathrm{Da}$, with no missed cleavages. A wide mass window was used to increase peptide identifications [16]. Modifications included in the searches are as follows: static modification of cysteine 57.021464 Da (C[143.13]), and variable modifications of methionine $15.994915 \mathrm{Da}$ $\mathrm{M}[147.19]$, glutamine -17.0306 Da Q[111.10], glutamic acid -18.01,056 Da E[111.10], lysine 8.014199 Da, K[136.19], and arginine 10.008269 Da R[166.20].

Individual search results were processed using the Trans Proteomic Pipeline [17]. Search result validation was done using Peptide Prophet (ver. 3.0) [18], and relative quantification of isotopically labeled peptides was reported by ASAP [19] and Xpress [20], both of which integrate the AUC of an ion chromatogram. Scores of $-1,0$, and 999 (no information) were excluded from the final distribution analyses. A probability of 0.9 was used as the cutoff for peptides. This cutoff returned a total of 17,318 spectra containing 124 decoy peptides for an overall false discovery rate of $0.7 \%$ for peptides.

\section{Post-Pipeline Analysis of MS/MS Data}

Tab-delimited Peptide Prophet text files were imported into the QTIPS application (available at: http://qtips. systemsbiology.net), which identifies informative peptides and calculates the frequency of heavy arginine or heavy lysine containing peptides in the population of informative peptides. Informative peptides are those that contain either a single $\mathrm{K}$ or a single $\mathrm{R}$, not both, which also meet user defined Peptide Prophet probability cut-offs (default $0.9,90 \%$ confidence), thresholding based on the total number of peptide identifications for each protein (default 0 , no threshold) and peptide ID search limits (default set to analyze yeast proteins only). The QTIPS application also determines the average Xpress and ASAP percent incorporation values for informative peptides and presents histograms of their distributions. Heat maps of ASAP and Xpress distributions were generated using MultiExperiment Viewer v4.4.

\section{Results and Discussion}

\section{Quantification of Heavy Amino Acid Incorporation} by Total Peptide Identifications

The relationship between global heavy amino acid incorporation and the relative number of heavy labeled peptides identified by MS/MS was investigated as an unbiased means to quantify heavy amino acid incorporation levels in biological samples (Supplemental Figure, which can be found in the electronic version of this article). As proof of principle for this method, termed QTIPS (Quantification by Total Identified Peptides for SILAC), we performed a simple mixing experiment in 

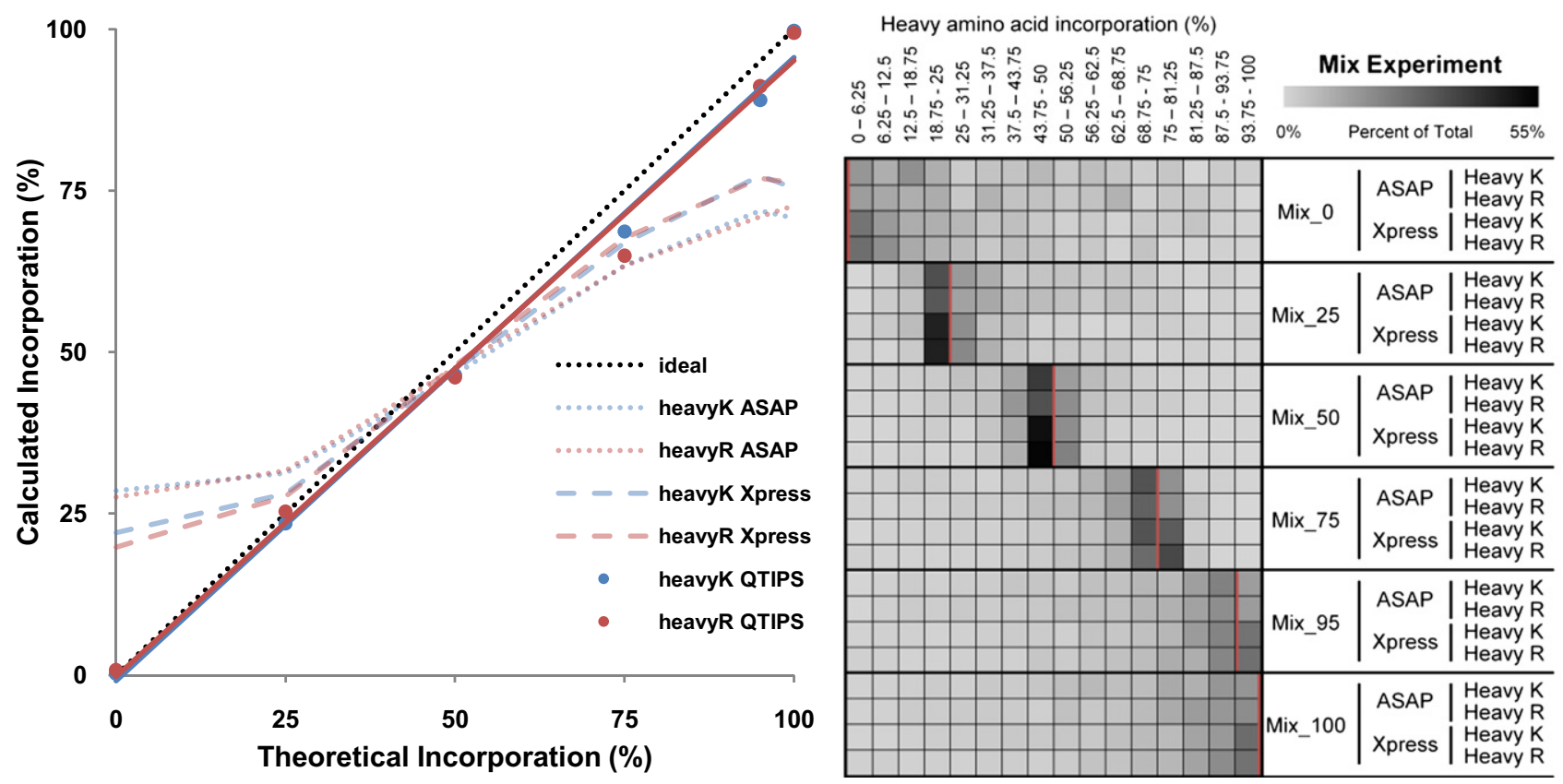

Figure 1. QTIPS is an accurate method to determine the extent of heavy amino acid incorporation in SILAC labeling experiments. (left) Samples of known heavy:light protein ratios were prepared and incorporation was calculated by QTIPS, ASAP, or Xpress. Incorporation rates derived by QTIPS for both heavy lysine and heavy arginine trend along the ideal plot (dotted line), indicating that they correlate well with theoretical values $\left(\mathrm{r}^{2}=0.996\right.$ and 0.991 , respectively). ASAP and Xpress estimates are inaccurate at low or high levels of incorporation. (right) Heat maps displaying the ASAP or Xpress derived distributions of heavy amino acid incorporation observed for lysine and arginine peptide identifications in each mix sample. Red lines indicate mean expected percent incorporation values.

which protein samples of known amounts of heavy arginine and lysine incorporation were analyzed and compared to the expected level of incorporation. Fully heavy labeled (10 generations in SILAC-KR media) and fully light labeled (10 generations in CSM) lysates were prepared and, from these, samples with heavy amino acid incorporation levels of $0 \%, 25 \%, 50 \%, 75 \%, 95 \%$, and $100 \%$ were prepared and analyzed by MS/MS. The results, (Figure 1), demonstrate the utility of the QTIPS method to accurately predict the level of heavy lysine and arginine in a protein sample over a wide range of incorporation. Importantly, an analysis of the same data using two established alternate methods, ASAP [19] and Xpress [20], clearly demonstrate their limitations when applied without manual curation to complex protein mixtures wherein one isotopic variant predominates (see also Table 1). ASAP and Xpress integrate AUC of MS1 traces for relative quantification. Both perform well within a 2 -fold relative abundance range, but become inaccurate at low or high heavy amino acid incorporation levels.

\section{SILAC Incorporation is Unperturbed by Intact Amino Acid Biosynthesis Pathways}

Most commonly, yeast SILAC experiments utilizing heavy arginine and lysine are performed in strains auxotrophic for these amino acids, eliminating the cell's inherent ability to synthesize light counterparts. This has the potential to be a major hurdle, given that many widely used laboratory strains and libraries are not auxotrophic for one or both of these amino acids; therefore, we used QTIPS to determine if efficient heavy amino acid incorporation in yeast requires auxotrophy.

We hypothesized that, in strains with intact lysine and arginine amino acid biosynthesis pathways, cells would preferentially utilize available free heavy lysine and arginine rather than spend resources on amino acid synthesis. To test this, the incorporation of heavy lysine and arginine was monitored by the QTIPS method in both LYS+ARG + and lys-arg- strains through nine generations. In agreement with previous findings $[1,6$, $9,10]$, near complete incorporation was achieved within seven generations for the lys-arg- strain and heavy amino acid incorporation in the prototrophic (LYS+ARG+) strain closely mirrored that observed in the dually auxotrophic background (Figure 2, Table 1). In both cases, full incorporation was achieved within seven generations. Relative to heavy lysine, there was no bias in the incorporation of heavy arginine and, therefore, it is unlikely that arginine-to-proline interconversion is a significant source of error at this number of doublings. Notably, all samples trend along the predicted incorporation based on population doubling, which suggests that the number of gener- 
Table 1. Summary of SILAC incorporation analysis by QTIPS, ASAP, and Xpress

\begin{tabular}{|c|c|c|c|c|c|c|}
\hline \multirow[b]{2}{*}{ Sample } & \multicolumn{6}{|c|}{ Mix experiment } \\
\hline & $0 \%$ & $25 \%$ & $50 \%$ & $75 \%$ & $95 \%$ & $100 \%$ \\
\hline Total peptide IDs & 1298 & 1326 & 1371 & 1408 & 1428 & 1120 \\
\hline Total protein IDs & 438 & 401 & 374 & 416 & 463 & 404 \\
\hline Cutoff peptides & 907 & 933 & 974 & 994 & 1022 & 782 \\
\hline Cutoff proteins & 287 & 258 & 243 & 267 & 320 & 290 \\
\hline Total K peptides & 434 & 452 & 461 & 472 & 510 & 372 \\
\hline Total R peptides & 391 & 411 & 436 & 436 & 430 & 347 \\
\hline Heavy K peptides & 1 & 106 & 214 & 324 & 454 & 371 \\
\hline Heavy R peptides & 3 & 104 & 201 & 283 & 392 & 345 \\
\hline QTIPS K incorp. (\%) & 0 & 23.5 & 46.4 & 68.6 & 89.0 & 99.7 \\
\hline QTIPS R incorp. (\%) & 1 & 25.3 & 46.1 & 64.9 & 91.2 & 99.4 \\
\hline Xpress $\mathrm{K}$ incorp. (\%) & 22.0 & 28.1 & 47.1 & 66.9 & 77.3 & 75.4 \\
\hline Xpress $\mathrm{R}$ incorp. (\%) & 19.8 & 27.6 & 47.9 & 67.6 & 76.9 & 76.2 \\
\hline ASAP $\mathrm{K}$ incorp (\%) & 28.5 & 31.2 & 46.5 & 63.4 & 71.9 & 70.7 \\
\hline ASAP R incorp (\%) & 27.5 & 31.7 & 47.6 & 63.4 & 70.9 & 72.6 \\
\hline False Discovery Rate & 0.007 & 0.004 & 0.003 & 0.006 & 0.003 & 0.001 \\
\hline Instrument & ORBI & ORBI & ORBI & ORBI & ORBI & ORBI \\
\hline
\end{tabular}

Sample: Theorectical \% heavy for 'Mix Experiment' and number of generations for 'Incorporation in LYS + ARG $+{ }^{\prime}$ and 'Incorporation in lys-arg-' Total peptide IDs and Total protein IDs: total number of unfiltered yeast peptide identifications from raw Peptide Prophet output files and their corresponding proteins; Cutoff peptides and Cutoff proteins: total number of yeast peptides meeting the probability cutoff of 0.9 ( $90 \%$ confidence) and their corresponding proteins; Total $\mathrm{K}$ peptides and Total $\mathrm{R}$ peptides: number of peptides from Cutoff peptides list containing a single $\mathrm{K}$ and not $\mathrm{R}$, or single $\mathrm{R}$ and not $\mathrm{K}$, respectively; Heavy $\mathrm{K}$ peptides and Heavy $\mathrm{R}$ peptides: number of peptides from Total $\mathrm{K}$ peptides list or Total $\mathrm{R}$ peptides list containing heavy $\mathrm{K}$ or heavy $\mathrm{R}$, respectively; QTIPS $\mathrm{K}$ incorp. $=100 \cdot($ Heavy $\mathrm{K}$ peptides)/(Total $\mathrm{K}$ peptides); $\mathrm{QTIPS} \mathrm{R}$ incorp. $=100 \cdot($ Heavy $\mathrm{R}$ peptides)/(Total R peptides); ASAP K incorp., ASAP R incorp., Xpress $\mathrm{K}$ incorp., and Xpress R incorp.: the L:H ASAP or Xpress ratios for data used in QTIPS analysis were converted to \%Heavy [\%Heavy $=100 /(1+\mathrm{L}:$ Hratio $)]$ from which the average \%Heavy values were calculated. False Discovery Rate: FDR = Decoy IDs/Total IDs at specified probability cut-off.

ations in SILAC media is the most important factor in determining incorporation levels in yeast. As with mixing experiments, we find QTIPS to be superior to unsupervised ASAP and Xpress in these complex protein mixtures (see also Table 1).

\section{Conclusion}

The QTIPS method that we have described is a simple, robust means of determining heavy isotope coded amino acid incorporation. It does not require manual
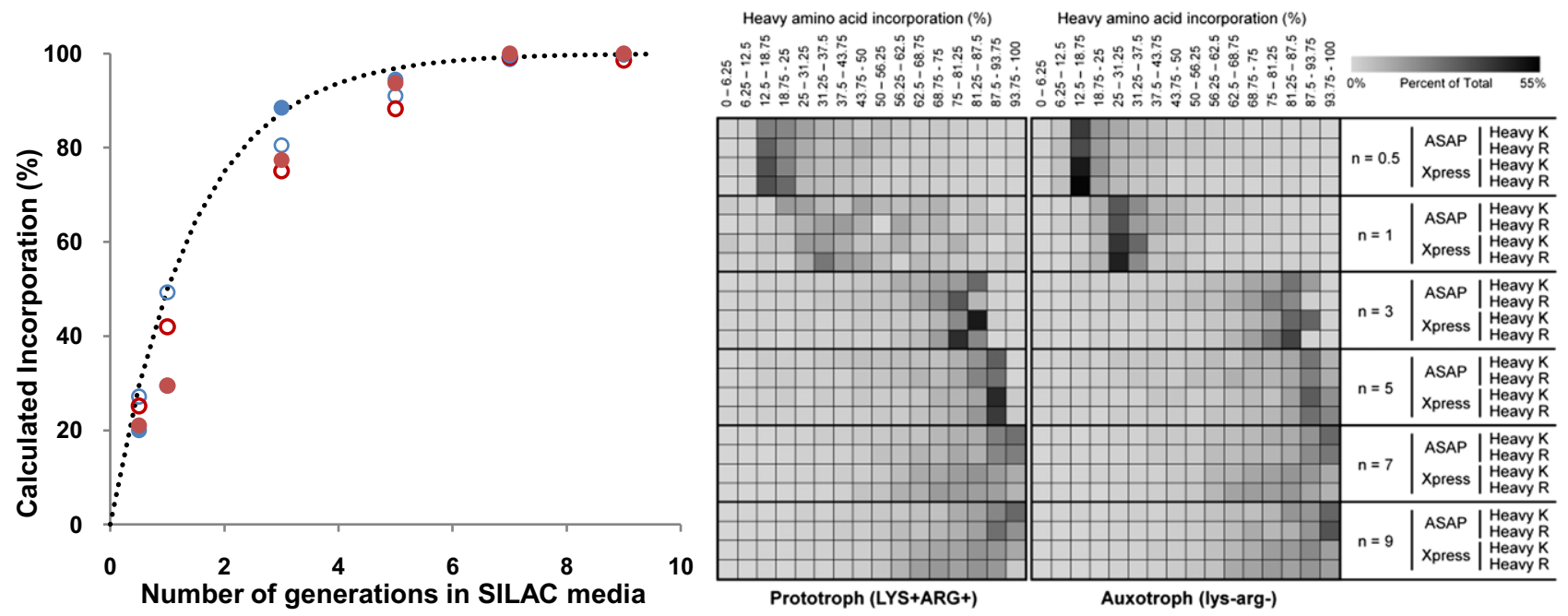

Figure 2. Auxotrophy is dispensable for efficient SILAC incorporation. (left) The level of heavy lysine incorporation in lys- (filled blue circle) and LYS+(open blue circle) strains and heavy arginine incorporation in arg- (filled red circle) and ARG+ (open red circle) strains were determined by the QTIPS method over a nine generation time-course. Regardless of genetic background, heavy amino acid incorporation reaches maximum levels after seven doublings. Incorporation models a cell division-based prediction of global protein synthesis during $\log$ phase growth $(\ldots \ldots \ldots)$. (right) Heat maps displaying the ASAP or Xpress derived distributions of heavy amino incorporation observed for lysine and arginine peptide identifications in each strain at each time point. 
Table 1. Continued

\begin{tabular}{|c|c|c|c|c|c|c|c|c|c|c|c|}
\hline \multicolumn{6}{|c|}{ Incorporation in LYS +ARG+ } & \multicolumn{6}{|c|}{ Incorporation in lys-arg- } \\
\hline 0.5 & 1 & 3 & 5 & 7 & 9 & 0.5 & 1 & 3 & 5 & 7 & 9 \\
\hline 1039 & 325 & 1520 & 1171 & 3117 & 2754 & 1302 & 1060 & 1460 & 1380 & 2983 & 3070 \\
\hline 332 & 191 & 464 & 419 & 869 & 751 & 374 & 405 & 420 & 447 & 839 & 854 \\
\hline 692 & 170 & 1046 & 789 & 1645 & 1522 & 913 & 653 & 1051 & 983 & 1607 & 1632 \\
\hline 173 & 83 & 233 & 217 & 306 & 274 & 201 & 185 & 231 & 253 & 302 & 261 \\
\hline 291 & 73 & 456 & 344 & 819 & 775 & 385 & 282 & 443 & 430 & 764 & 794 \\
\hline 310 & 81 & 457 & 341 & 682 & 607 & 409 & 278 & 464 & 430 & 684 & 668 \\
\hline 79 & 36 & 367 & 313 & 809 & 773 & 77 & 83 & 392 & 406 & 761 & 793 \\
\hline 78 & 34 & 343 & 301 & 676 & 598 & 86 & 82 & 359 & 403 & 684 & 668 \\
\hline 27.1 & 49.3 & 80.5 & 91.0 & 98.8 & 99.7 & 20.0 & 29.4 & 88.5 & 94.4 & 99.6 & 99.9 \\
\hline 25.2 & 42.0 & 75.1 & 88.3 & 99.1 & 98.5 & 21.0 & 29.5 & 77.4 & 93.7 & 100.0 & 100.0 \\
\hline 30.3 & 46.5 & 74.1 & 76.6 & 74.9 & 75.3 & 25.3 & 34.9 & 77.3 & 77.3 & 74.7 & 76.8 \\
\hline 29.4 & 46.7 & 72.2 & 76.1 & 74.0 & 74.0 & 24.5 & 34.6 & 73.1 & 76.8 & 73.9 & 77.0 \\
\hline 33.4 & 45.2 & 67.9 & 70.7 & 79.1 & 80.4 & 27.6 & 37.1 & 70.8 & 70.6 & 78.4 & 79.9 \\
\hline 32.1 & 50.4 & 66.1 & 71.6 & 78.2 & 78.5 & 29.4 & 37.1 & 67.1 & 71.7 & 77.4 & 81.3 \\
\hline 0.010 & 0.035 & 0.011 & 0.020 & 0.001 & 0.004 & 0.011 & 0.010 & 0.008 & 0.007 & 0.004 & 0.006 \\
\hline ORBI & ORBI & ORBI & ORBI & LTQ & LTQ & ORBI & ORBI & ORBI & ORBI & LTQ & LTQ \\
\hline
\end{tabular}

curation of MS quantification data and can be applied to both simple and complex protein mixtures. The accuracy and dynamic range of measurements by QTIPS depend directly on sample size (the total number of peptide identifications). Importantly, because the method includes all detected proteins, there is less likelihood that bias is introduced by variation in protein turnover rates, which can be an issue for MS1 quantification methods in which only a limited number of peptides and/or proteins are interrogated. We have focused our initial characterization of QTIPS using baker's yeast, but this methodology is applicable to any other experimentally manipulable biological system. In yeast, the incorporation of heavy amino acids is highly efficient in strains with intact amino acid biosynthesis pathways, making the method suitable for quantitative proteomics without the need for additional genetic manipulations. The potential of isotopic pulse-chase to estimate turnover of individual proteins on a global scale has been demonstrated [11], but is limited by the time required for manual data curation. The combination of QTIPS with large MS/MS datasets allows quantification at single protein resolution, and is widely applicable to methodologies exploiting isotopic labeling for quantitative mass spectrometry-based proteomics.

\section{Acknowledgments}

The authors acknowledge support for this work by grants GM075152, GMO76547, and RR022220 from the U.S. National Institutes of Health.

\section{Appendix A Supplementary Material}

Supplementary material associated with this article may be found in the online version at doi:10.1016/ j.jasms.2010.04.002. The QTIPS application is available at http://qtips.systemsbiology.net/.

\section{References}

1. Ong, S. E.; Blagoev, B.; Kratchmarova, I.; Kristensen, D. B.; Steen, H.; Pandey, A.; Mann, M. Stable Isotope Labeling by Amino Acids in Cell Culture SILAC as a Simple and Accurate Approach to Expression Proteomics. Mol. Cell. Proteom. 2002, 1, 376-386.

2. Pan, C.; Kumar, C.; Bohl, S.; Klingmueller, U.; Mann, M. Comparative Proteomic Phenotyping of Cell Lines and Primary Cells to Assess Preservation of Cell Type-Specific Functions. Mol. Cell. Proteom. 2009, 8, 443-450.

3. Mann, M. Functional and Quantitative Proteomics Using SILAC. Nat. Rev. Mol. Cell Biol. 2006, 7, 952-958.

4. Kruger, M.; Moser, M.; Ussar, S.; Thievessen, I.; Luber, C. A.; Forner, F.; Schmidt, S.; Zanivan, S.; Fassler, R.; Mann, M. SILAC Mouse for Quantitative Proteomics Uncovers Kindlin-3 as an Essential Factor for Red Blood Cell Function. Cell 2008, 134, 353-364.

5. Ong, S. E.; Kratchmarova, I.; Mann, M. Properties of 13C-Substituted Arginine in Stable Isotope Labeling by Amino Acids in Cell Culture (SILAC). I. Proteome Res. 2003, 2, 173-181.

6. Schmidt, F.; Strozynski, M.; Salus, S. S.; Nilsen, H.; Thiede, B. Rapid Determination of Amino Acid Incorporation by Stable Isotope Labeling with Amino Acids in Cell Culture (SILAC). Rapid Commun. Mass Spectrom. 2007, 21, 3919-3926.

7. Van Hoof, D.; Pinkse, M. W.; Oostwaard, D. W.; Mummery, C. L.; Heck, A. J.; Krijgsveld, J. An Experimental Correction for Arginine-to-Proline Conversion Artifacts in SILAC-Based Quantitative Proteomics. Nat. Methods 2007, 4, 677-678.

8. Gruhler, A.; Olsen, J. V.; Mohammed, S.; Mortensen, P.; Faergeman, N. J.; Mann, M.; Jensen, O. N. Quantitative Phosphoproteomics Applied to the Yeast Pheromone Signaling Pathway. Mol. Cell. Proteom. 2005, 4, 310-327.

9. Ong, S. E.; Mann, M. A Practical Recipe for Stable Isotope Labeling by Amino Acids in Cell Culture (SILAC). Nat. Prot. 2006, 1, 2650-2660. 
10. Ong, S. E.; Mann, M. Stable Isotope Labeling by Amino Acids in Cell Culture for Quantitative Proteomics. Methods Mol. Biol. 2007, 359, 37-52.

11. Pratt, J. M.; Petty, J.; Riba-Garcia, I.; Robertson, D. H.; Gaskell, S. J.; Oliver, S. G.; Beynon, R. J. Dynamics of Protein Turnover, a Missing Dimension in Proteomics. Mol. Cell. Proteom. 2002, 1, 579-591.

12. Belle, A.; Tanay, A.; Bitincka, L.; Shamir, R.; O'Shea, E. K. Quantification of Protein Half-Life in the Budding Yeast Proteome. Proc. Natl. Acad. Sci. U.S.A. 2006, 103, 13004-13009.

13. Saleem, R.; Rogers, R.; Ratushny, A.; Dilworth, D.; Shannon, P.; Shteynberg, D.; Wan, Y.; Moritz, R.; Nesvizhskii, A.; Rachubinski, R.; Aitchison, J. Integrated Phosphoproteomic Analysis of a Signaling Network Governing Nutrient Response and Peroxisome Induction. Mol. Cell. Proteom. First Published on April 15, 2010, doi: 10.1074/mcp.M000116MCP201.

14. Craig, R.; Beavis, R. C. TANDEM: Matching Proteins with Tandem Mass Spectra. Bioinformatics 2004, 20, 1466-1467.

15. MacLean, B.; Eng, J. K.; Beavis, R. C.; McIntosh, M. General Framework for Developing and Evaluating Database Scoring Algorithms
Using the TANDEM Search Engine. Bioinformatics 2006, 22, 2830 2832

16. Hsieh, E. J.; Hoopmann, M. R.; MacLean, B.; MacCoss, M. J. Comparison of Database Search Strategies for High Precursor Mass Accuracy MS/MS Data. J. Protein Res. 2010, 9, 1138-1143.

17. Keller, A.; Eng, J.; Zhang, N.; Li, X. J.; Aebersold, R. A Uniform Proteomics MS/MS Analysis Platform Utilizing Open XML File Formats. Mol. Sys. Biol. 2005, 1, 20050017.

18. Keller, A.; Nesvizhskii, A. I.; Kolker, E.; Aebersold, R. Empirical Statistical Model to Estimate the Accuracy of Peptide Identifications Made by MS/MS and Database Search. Anal. Chem. 2002, 74, 5383-5392.

19. Li, X. J.; Zhang, H.; Ranish, J. A.; Aebersold, R. Automated Statistical Analysis of Protein Abundance Ratios from Data Generated by Stable-Isotope Dilution and Tandem Mass Spectrometry. Anal. Chem. 2003, 75, 6648-6657.

20. Han, D. K.; Eng, J.; Zhou, H.; Aebersold, R. Quantitative Profiling of Differentiation-Induced Microsomal Proteins Using Isotope-Coded Affinity Tags and Mass Spectrometry. Nat. Biotechnol. 2001, 19, 946-951. 